\title{
Regulation of Myocardial Amino Acid Balance in the Conscious Dog
}

Ronald G. Schwartz, Eugene J. Barrett, Charles K. Francis, Ralph Jacob, and Barry L. Zaret

Department of Medicine, Cardiology and Endocrine Sections, Yale University School of Medicine, New Haven, Connecticut 06510

\section{Abstract}

The effects in vivo of physiologic increases in insulin and amino acids on myocardial amino acid balance were evaluated in conscious dogs. Arterial and coronary sinus concentrations of amino acids and coronary blood flow were measured during a 30-min basal and a 100-min experimental period employing three protocols: euglycemic insulin clamp (plasma insulin equaled $70 \pm 11 \mu \mathrm{U} / \mathrm{ml}, n=6$ ); euglycemic insulin clamp during amino acid infusion (plasma insulin equaled $89 \pm 12 \mu \mathrm{U} / \mathrm{ml}, n$ $=6$ ); and suppression of insulin with somatostatin during amino acid infusion (plasma insulin equaled $15 \pm 4 \mu \mathrm{U} / \mathrm{ml}, n$ =6).

Basally, only leucine and isoleucine were removed signifcantly by myocardium (net branched chain amino acid [BCAA] uptake equaled $0.5 \pm 0.2 \mu \mathrm{mol} / \mathrm{min}$ ), while glycine, alanine, and glutamine were released. Glutamine demonstrated the highest net myocardial production (1.6 $\pm 0.2 \mu \mathrm{mol} / \mathrm{min})$. No net exchange was seen for valine, phenylalanine, tyrosine, cysteine, methionine, glutamate, asparagine, serine, threonine, taurine, and aspartate. In group I, hyperinsulinemia caused a decline of all plasma amino acids except alanine; alanine balance switched from release to an uptake of $0.6 \pm 0.4 \mu \mathrm{mol} / \mathrm{min}(P<0.05)$, while the myocardial balance of other amino acids was unchanged. In group II, amino acid concentrations rose, and were accompanied by a marked rise in myocardial BCAA uptake $(0.4 \pm 0.1-2.6 \pm 0.3 \mu \mathrm{mol} / \mathrm{min}, P<0.001)$. Uptake of alanine was again stimulated $(0.9 \pm 0.3 \mu \mathrm{mol} / \mathrm{min}, P<0.01)$, while glutamine production was unchanged $(1.3 \pm 0.4$ vs. $1.6 \pm 0.3$ $\mu \mathrm{mol} / \mathrm{min})$. In group III, there was a 4-5-fold increase in the plasma concentration of the infused amino acids, accompanied by marked stimulation in uptake of only BCAA $(6.8 \pm 0.7 \mu \mathrm{mol} /$ min). Myocardial glutamine production was unchanged (1.9 $\pm 0.4-1.3 \pm 0.7 \mu \mathrm{mol} / \mathrm{min})$. Within the three experimental groups there were highly significant linear correlations between myocardial uptake and arterial concentration of leucine, isoleucine, valine, and total BCAA $(r=0.98,0.98,0.92$, and 0.97 , respectively); $P<0.001$ for each).

In vivo, BCAA are the principal amino acids taken up by the myocardium basally and during amino acid infusion. Plasma BCAA concentration and not insulin determines the rate of myocardial BCAA uptake. Insulin stimulates myocardial alanine uptake. Neither insulin nor amino acid infusion alters myocardial glutamine release.

Address correspondence to Dr. Schwartz, Cardiology Section.

Received for publication 15 August 1984 and in revised form 18 December 1984.

J. Clin. Invest.

(C) The American Society for Clinical Investigation, Inc.

0021-9738/85/04/1204/08 $\$ 1.00$

Volume 75, April 1985, 1204-1211

\section{Introduction}

Fatty acids, glucose, and lactate are the principal oxidative fuels in myocardium. Amino acids serve a minor role as an oxidative fuel (1-3) and a more essential role as substrate for protein synthesis (4). Studies of isolated perfused rodent hearts suggest that myocardial use of amino acids for protein synthesis may be regulated by $(a)$ the availability of amino acids (3-6); (b) the supply of oxidative substrates (3); (c) the adequacy of oxygen delivery (3); $(d)$ the availability of hormones, such as insulin $(3,5)$; and $(e)$ the level of ventricular pressure development (3). In both cardiac and skeletal muscle, the addition in vitro of insulin or a physiologic concentration of amino acids to the perfusate stimulates synthesis and inhibits degradation of protein $(4,7)$. In the isolated perfused rat heart, synthesis of whole heart protein and myosin is increased when amino acid levels in the perfusate are increased to five times normal plasma levels (4). However, addition of insulin to the perfusate containing glucose and normal plasma levels of amino acids accelerates the initiation of peptide chains more effectively than addition of amino acids at five times normal plasma levels $(3,5)$. Furthermore, in the presence of insulin, high levels of amino acids have no further stimulating effect on the rate of synthesis (5). Myocardial protein degradation is inhibited by amino acids in the perfusate, and branched chain amino acids (BCAA) ${ }^{1}$ mimic the effect of the complete mixture (3). As with protein synthesis, insulin has been found as well to be a more potent inhibitor of protein degradation than have increased perfusate concentrations of amino acids alone (3).

In contrast to these in vitro data, evidence that in vivo insulin administration or deficiency affects myocardial amino acid metabolism or protein synthesis is scant. Pain and Garlick (8) have reported an accelerated fractional turnover of heart protein in insulin-deficient diabetic animals, which was corrected by insulin administration. Unfortunately, the rates of amino acid utilization and of protein synthesis could not be obtained directly from their data.

Using classical organ balance techniques, conflicting data have been reported for postabsorptive myocardial amino acid balance in man. Carlsten et al. (9) found that in healthy, nonhospitalized volunteers, there was no net myocardial uptake or release of amino acids other than for the release of alanine. Mudge et al. (10) examined myocardial amino acid exchange in patients undergoing cardiac catheterization because of chest pain syndromes. They reported myocardial uptake of glutamate at rest and release of alanine with pacing in eight patients who did not have angiographically demonstrable coronary artery disease. No significant arterio-coronary sinus concentration differences were found for the other amino acids. In 11 patients

1. Abbreviation used in this paper: BCAA, branched chain amino acid(s). 
with coronary artery disease, glutamate uptake and alanine release were found both in the resting state and during pacing to sub-anginal threshold rates. In contrast, Brodan et al. observed markedly positive myocardial uptake of only aspartate at rest, and uptake of glutamate, leucine, and isoleucine, and release of cystine-cysteine, glutamine, and asparagine during pacing in nine patients with coronary artery disease (11). Each of these studies examined cardiac amino acid balance in the postabsorptive state and neither the role of insulin nor of plasma amino acid concentration was determined.

In the present study we used the insulin clamp technique $(12,13)$ in combination with intravenous infusion of amino acids and arterial and coronary sinus blood sampling to study the effect of physiologic changes in plasma insulin and/or amino acids on myocardial balance of amino acids. Clarification of the in vivo quantitative relationships between plasma concentrations of insulin and amino acids and uptake of amino acids remains important from a physiologic standpoint. In addition, further interest is stimulated from recent reports that glutamate (14-18), aspartate $(15,16)$, and the BCAA (19) may preserve contractile function in oxygen-deprived myocardium, and that labeled synthetic BCAA (20) and glutamate (21) may be suitable for in vivo positron emission tomographic imaging of regional myocardial metabolism.

\section{Methods}

Animal preparation. $24-48 \mathrm{~h}$ before study, adult mongrel dogs of either sex weighing between 19.1 and $30.9 \mathrm{~kg}$ (mean $=22.9 \pm 0.7 \mathrm{~kg}$ ) were anesthetized with sodium pentabarbital. Under aseptic conditions, an incision was made in the right side of the neck and the carotid artery and jugular vein were isolated. A heparin-filled silastic catheter (1.57 $\mathrm{mm}$ inner diameter, Dow Corning Corp., Midland, MI) was placed in each vessel, and the extravascular ends occluded and fixed in the subcutaneous tissue. The overlying skin was sutured closed. On the day of study, after an overnight fast, the awake animal was gently restrained on a fluoroscopy table in the left lateral decubitus position. After administration of $1 \%$ lidocaine locally, the sutures were removed from the neck and the free ends of the catheters exposed. The arterial catheter was used for obtaining arterial blood samples and for monitoring blood pressure and heart rate. The second silastic catheter was used as a retaining catheter, and was removed at the time of study and replaced with a triple thermistor thermodilution catheter (Webster Laboratories, Inc., Altadena, CA) which then was positioned under fluoroscopic guidance with the proximal thermistor in the main portion of the coronary sinus. A 7F Sones catheter (USCI, Billerica, MA) was also advanced into the main portion of the coronary sinus and was used for sampling. An 18-gauge polyethylene catheter was placed percutaneously in the right saphenous vein and used for infusion of hormones and substrates. Patency of all catheters was maintained by slow saline infusion. Position of the coronary sinus catheters was confirmed at the beginning and end of each experiment by fluoroscopy and measurement of oxygen saturation in blood samples.

Experimental protocols. Coronary sinus blood flow and arterial and coronary sinus plasma concentrations of glucose and amino acids and arterial concentrations of insulin were measured every 10 min during a 30-min basal period, and every 20 min during a 100 -min study period. Six dogs were studied in each group. In group I, the euglycemic insulin clamp was employed. After obtaining basal samples, plasma insulin was raised $\sim 60 \mu \mathrm{U} / \mathrm{ml}$ above fasting levels using a primed (2 $\mathrm{mU} / \mathrm{min} \cdot \mathrm{kg} \times 10 \mathrm{~min})$ continuous $(1 \mathrm{mU} / \mathrm{min} \cdot \mathrm{kg} \times 90 \mathrm{~min})$ infusion of regular insulin (Eli Lilly \& Co., Indianapolis, IN). Plasma glucose was maintained within $10 \%$ of basal values by measuring plasma glucose every $5 \mathrm{~min}$ and adjusting the rate of infusion of a $20 \%$ dextrose solution as previously described $(12,13)$.
In group II, after the basal period, insulin was again given, and plasma glucose was maintained at basal levels as in group I. In addition, to prevent the decline in plasma amino acids seen in the group I, a $10 \%$ amino acid hydrolysate mixture (Travasol, Travenol Co., Deerfield, IL) was infused at a priming rate of $15.5 \mathrm{mg} / \mathrm{kg} \cdot \min$ for the first 10 $\min$ and then at a constant rate of $7.73 \mathrm{mg} / \mathrm{kg} \cdot \min$ for $90 \mathrm{~min}$.

In group III, after the 30-min basal period, somatostatin $(500 \mu \mathrm{g} /$ h) was infused to inhibit endogenous insulin secretion. The amino acid infusion was identical to that used in group II; however, no insulin or glucose was infused in this group.

Chemical determinations. Plasma glucose was measured using the glucose oxidase method (22) with a Beckman glucose analyzer (Beckman Instruments, Inc., Palo Alto, CA). Plasma insulin was assayed as described previously (13). Amino acid determinations were made using a Dionex D-500 (Dionex Corp., Sunnyvale, CA) automated amino acid analyzer, with an L6 column and lithium citrate buffers. The basic amino acids, histidine, lysine, and arginine were not quantified in the current analysis.

Calculations. Coronary sinus blood flow was calculated from thermodilution flow curves as described by Ganz et al. (23) and validated by Pepine et al. (24). An analogue to digital converter and a computer (Hewlett-Packard model 9835A, Hewlett-Packard Co., Palo Alto, CA) were used for rapid calculation of the coronary blood flow from the thermodilution flow curves. Myocardial amino acid exchange was calculated from the product of the arterial-coronary sinus plasma concentration difference times the coronary plasma flow.

Statistical analysis of the change in myocardial amino acid exchange over time and comparison among experimental groups was performed using either paired or unpaired $t$ test or analysis of variance with a repeated measures design (25). Data are expressed as mean \pm SEM.

\section{Results}

During the basal period we observed a significant arterialcoronary sinus plasma concentration gradient for five amino acids. The net myocardial balances of these amino acids are shown in Fig. 1. Only leucine and isoleucine showed a significant uptake in the myocardium. The sum of the BCAA uptake (leucine plus isoleucine plus valine) averaged $0.5 \pm 0.2$

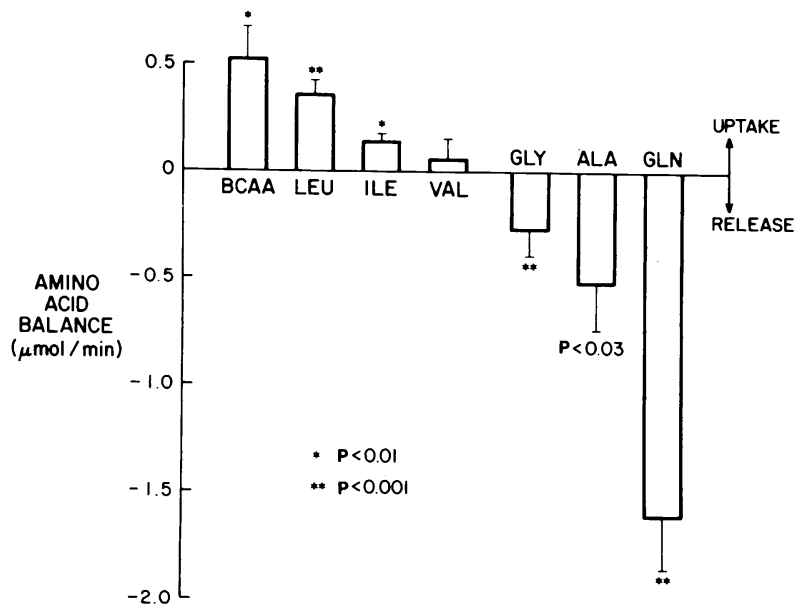

Figure 1. Myocardial net balance of amino acids in the basal state is illustrated in 18 conscious, normal mongrel dogs. Myocardial uptake is indicated by the bars above the line, and net release of amino acids is represented by the bars below the line. Error bars indicate \pm SEM. Degrees of significance reflect differences in arterial/coronary sinus concentrations of the amino acid ( $P$ values from paired $t$ test). LEU, leucine; ILE, isoleucine; VAL, valine; GLY, glycine; ALA, alanine; GLN, glutamine. 


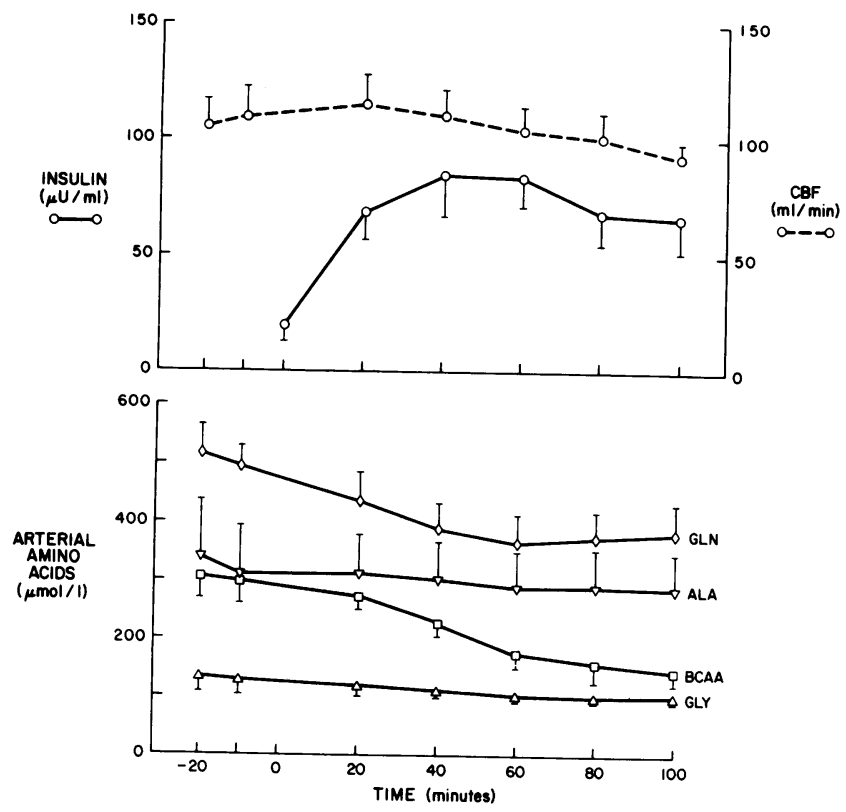

Figure 2. Arterial concentrations of insulin and amino acids and coronary blood (CBF) flow during insulin and glucose infusion in group I dogs $(n=6)$. (Top) Plasma insulin concentrations are plotted with open circles that are connected by the solid line, and coronary blood flow is plotted with the open circles and the dashed line. (Bottom) Arterial plasma concentrations of amino acids during the insulin clamp. Abbreviations are as designated in Fig. 1.

$\mu \mathrm{mol} / \mathrm{min}$. Glycine, alanine, and glutamine were released by the myocardium. Glutamine demonstrated the highest net myocardial production $(1.6 \pm 0.2 \mu \mathrm{mol} / \mathrm{min})$. Net basal exchange for the other 11 amino acids analyzed (valine, phenylalanine, tyrosine, cysteine, methionine, glutamate, asparagine, serine, threonine, aspartate, and taurine) was not significantly different from zero. No differences in myocardial amino acid balances were found among the three groups of dogs in the basal state. There was a small uptake of glucose $1.6 \pm 0.4 \mu \mathrm{mol} / \mathrm{min}$ ) by the myocardium in the basal state. Coronary blood flow during the postabsorptive period averaged $101 \pm 8 \mathrm{ml} / \mathrm{min}$.

Infusion of insulin and glucose in group I dogs $(n=6)$ raised plasma insulin by $53 \pm 10 \mu \mathrm{U} / \mathrm{ml}$, while arterial glucose was maintained within $10 \%$ of the postabsorptive values $(98 \pm 6$ $\mathrm{mg} / \mathrm{dl})$. Myocardial glucose uptake increased to $21.3 \pm 5.0$ $\mu \mathrm{mol} / \mathrm{min}$ during the last $40 \mathrm{~min}$ of insulin infusion $(P$ $<0.002$ ). Coronary blood flow did not change significantly during the study period (Fig. 2). The arterial concentrations of all amino acids except alanine declined by $20-50 \%$ over the first $60 \mathrm{~min}$ and maintained a stable concentration between 60 and $100 \mathrm{~min}$ of the infusion protocol (Table I, Fig. 2). Myocardial uptake of each of the BCAA tended to decline, as did the release of glycine and glutamine; however, these changes were not significant (Table II). In contrast, basal alanine efflux was reversed, with significant net uptake during insulin infusion. Over the 100 -min experimental period, the myocardial balance of other amino acids did not change significantly from basal.

In group II, a rise in plasma insulin of $69 \pm 12 \mu \mathrm{U} / \mathrm{ml}(P$ $=$ NS vs. group I; Fig. 3) was noted. Coronary blood flow during the insulin infusion did not change compared to baseline $(77 \pm 11$ vs. $91 \pm 7 \mathrm{ml} / \mathrm{min})$. Glucose was maintained at basal levels $(97 \pm 3 \mathrm{mg} / \mathrm{dl})$ while myocardial glucose uptake increased to $6.4 \pm 3.9 \mu \mathrm{mol} / \mathrm{min}$ during the last $40 \mathrm{~min}$ of infusion. There was a 2-4-fold increase in the arterial levels of each of the BCAA, glycine, and alanine (Table I; Fig. 3). The plasma concentrations of other infused amino acids rose

Table I. Concentrations of Amino Acids in Arterial Plasma

\begin{tabular}{|c|c|c|c|c|c|c|}
\hline \multirow[b]{2}{*}{ AA } & \multicolumn{2}{|l|}{ Group I } & \multicolumn{2}{|l|}{ Group II } & \multicolumn{2}{|l|}{ Group III } \\
\hline & Basal & $60-100 \mathrm{~min}$ & Basal & $60-100 \mathrm{~min}$ & Basal & $60-100 \mathrm{~min}$ \\
\hline \multirow[t]{2}{*}{ BCAA } & $290 \pm 30$ & $149 \pm 22$ & $271 \pm 24$ & $501 \pm 30$ & $323 \pm 29$ & $1,215 \pm 211$ \\
\hline & \multicolumn{2}{|c|}{$P<0.01$} & \multicolumn{2}{|c|}{$P<0.001$} & \multicolumn{2}{|c|}{$P<0.001$} \\
\hline \multirow[t]{2}{*}{ LEU } & $104 \pm 11$ & $47 \pm 9$ & $96 \pm 9$ & $159 \pm 11$ & $118 \pm 12$ & $396 \pm 31$ \\
\hline & \multicolumn{2}{|c|}{$P<0.01$} & \multicolumn{2}{|c|}{$P<0.001$} & \multicolumn{2}{|c|}{$P<0.001$} \\
\hline \multirow[t]{2}{*}{ ILE } & $46 \pm 5$ & $19 \pm 4$ & $40 \pm 5$ & $105 \pm 8$ & $44 \pm 6$ & $265 \pm 15$ \\
\hline & \multicolumn{2}{|c|}{$P<0.002$} & \multicolumn{2}{|c|}{$P<0.001$} & \multicolumn{2}{|c|}{$P<0.001$} \\
\hline \multirow[t]{2}{*}{ VAL } & $141 \pm 13$ & $83 \pm 9$ & $135 \pm 12$ & $247 \pm 17$ & $156 \pm 15$ & $554 \pm 42$ \\
\hline & \multicolumn{2}{|c|}{$P<0.001$} & \multicolumn{2}{|c|}{$P<0.001$} & \multicolumn{2}{|c|}{$P<0.001$} \\
\hline \multirow[t]{2}{*}{ GLY } & $1,217 \pm 18$ & $91 \pm 8$ & $106 \pm 11$ & $665 \pm 40$ & $103 \pm 9$ & $673 \pm 27$ \\
\hline & \multicolumn{2}{|c|}{$P=0.07$} & \multicolumn{2}{|c|}{$P<0.001$} & \multicolumn{2}{|c|}{$P<0.001$} \\
\hline \multirow[t]{2}{*}{ ALA } & $314 \pm 186$ & $276 \pm 126$ & $139 \pm 13$ & $671 \pm 51$ & $204 \pm 26$ & $1,019 \pm 36$ \\
\hline & \multicolumn{2}{|c|}{$P$ NS } & \multicolumn{2}{|c|}{$P<0.001$} & \multicolumn{2}{|c|}{$P<0.001$} \\
\hline \multirow[t]{2}{*}{ GLN } & $495 \pm 39$ & $362 \pm 43$ & $449 \pm 39$ & $328 \pm 18$ & $377 \pm 25$ & $529 \pm 27$ \\
\hline & \multicolumn{2}{|c|}{$P<0.01$} & \multicolumn{2}{|c|}{$P<0.02$} & \multicolumn{2}{|c|}{$P<0.001$} \\
\hline
\end{tabular}

Basal and stimulated (meaned 60-100 min) arterial plasma concentrations of amino acids for which a net myocardial flux was found. Values are expressed as micromoles per liter (mean \pm SEM). $P$ values reflect comparisons using paired $t$ tests of meaned basal vs. stimulated values for each amino acid. LEU, leucine; ILE, isoleucine; VAL, valine; GLY, glycine; ALA, alanine; GLN, glutamine; NS, not significant. 
Table II. Myocardial Balance of Amino Acids

\begin{tabular}{|c|c|c|c|c|c|c|}
\hline \multirow[b]{2}{*}{ AA } & \multicolumn{2}{|l|}{ Group I } & \multicolumn{2}{|l|}{ Group II } & \multicolumn{2}{|l|}{ Group III } \\
\hline & Basal & $60-100 \mathrm{~min}$ & Basal & $60-100 \mathrm{~min}$ & Basal & $60-100 \mathrm{~min}$ \\
\hline \multirow[t]{2}{*}{ BCAA } & $0.6 \pm 0.2$ & $0.2 \pm 0.1$ & $0.4 \pm 0.1$ & $2.6 \pm 0.3$ & $0.5 \pm 0.4$ & $6.8 \pm 0.7$ \\
\hline & \multicolumn{2}{|c|}{$P$ NS } & \multicolumn{2}{|c|}{$P<0.002$} & \multicolumn{2}{|c|}{$P<0.001$} \\
\hline \multirow[t]{2}{*}{ LEU } & $0.35 \pm 0.09$ & $0.12 \pm 0.06$ & $0.24 \pm 0.04$ & $1.0 \pm 0.2$ & $0.5 \pm 0.1$ & $2.7 \pm 0.3$ \\
\hline & \multicolumn{2}{|c|}{$P \mathrm{NS}$} & \multicolumn{2}{|c|}{$P<0.01$} & \multicolumn{2}{|c|}{$P<0.01$} \\
\hline \multirow[t]{2}{*}{ ILE } & $0.17 \pm 0.06$ & $0.04 \pm 0.06$ & $0.09 \pm 0.03$ & $0.7 \pm 0.1$ & $0.14 \pm 0.06$ & $2.1 \pm 0.2$ \\
\hline & \multicolumn{2}{|c|}{$P$ NS } & \multicolumn{2}{|c|}{$P<0.002$} & \multicolumn{2}{|c|}{$P<0.001$} \\
\hline \multirow[t]{2}{*}{ VAL } & $0.08 \pm 0.08$ & $0.02 \pm 0.08$ & $0.12 \pm 0.08$ & $0.87 \pm 0.08$ & $-0.03 \pm 0.25$ & $2.0 \pm 0.3$ \\
\hline & \multicolumn{2}{|c|}{$P$ NS } & \multicolumn{2}{|c|}{$P<0.01$} & \multicolumn{2}{|c|}{$P<0.01$} \\
\hline \multirow[t]{2}{*}{ GLY } & $-0.7 \pm 0.2$ & $-0.3 \pm 0.1$ & $-0.1 \pm 0.04$ & $-0.9 \pm 0.6$ & $-0.1 \pm 0.2$ & $0.6 \pm 0.4$ \\
\hline & \multicolumn{2}{|c|}{$P$ NS } & \multicolumn{2}{|c|}{$P$ NS } & \multicolumn{2}{|c|}{$P$ NS } \\
\hline \multirow[t]{2}{*}{ ALA } & $-0.8 \pm 0.6$ & $0.6 \pm 0.4$ & $-0.3 \pm 0.1$ & $0.9 \pm 0.3$ & $-0.5 \pm 0.2$ & $1.2 \pm 0.8$ \\
\hline & \multicolumn{2}{|c|}{$P<0.05$} & \multicolumn{2}{|c|}{$P=0.01$} & \multicolumn{2}{|c|}{$P$ NS } \\
\hline \multirow[t]{2}{*}{ GLN } & $-1.6 \pm 0.4$ & $-0.8 \pm 0.5$ & $-1.3 \pm 0.4$ & $-1.6 \pm 0.3$ & $-1.9 \pm 0.4$ & $-1.3 \pm 0.7$ \\
\hline & \multicolumn{2}{|c|}{$P$ NS } & \multicolumn{2}{|c|}{$P$ NS } & \multicolumn{2}{|c|}{$P$ NS } \\
\hline
\end{tabular}

Basal and stimulated (meaned 60-100 min) uptake of the amino acids for which a significant net myocardial flux was found. Values are expressed in micromoles per minute (mean \pm SEM). $P$ values reflect comparisons using paired $t$ tests of meaned basal vs. stimulated values for each amino acid. LEU, leucine; ILE, isoleucine; VAL, valine; GLY, glycine; ALA, alanine; GLN, glutamine; NS, not significant.

similarly. The arterial concentration of glutamine, an amino acid not in the amino acid infusate, fell by $25 \%$. The myocardial uptake of total and individual BCAA markedly increased (total BCAA: $0.4 \pm 0.1-2.6 \pm 0.3 \mu \mathrm{mol} / \mathrm{min}, P<0.002$ ) (Table II). Alanine uptake was stimulated to $0.9 \pm 0.3 \mu \mathrm{mol} / \mathrm{min}$, a level comparable to that found in group I. In contrast, glutamine

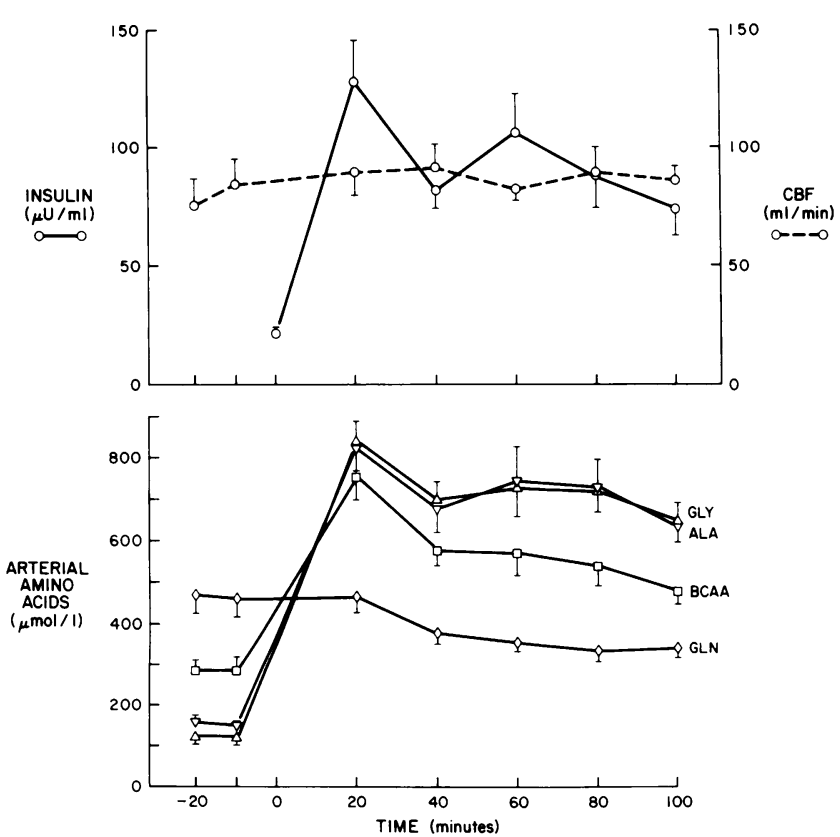

Figure 3. Arterial concentrations of insulin and amino acids and coronary blood flow (CBF) during insulin, glucose, and amino acid infusion in group II dogs $(n=6)$. Abbreviations are as designated in Fig. 1. production in this group $(1.6 \pm 0.3 \mu \mathrm{mol} / \mathrm{min})$ was unchanged when compared both with basal in this group and to the stimulated state in group I dogs.

In group III, somatostatin infusion resulted in a fall in insulin levels of $25 \%(20.3 \pm 4.4 \mu \mathrm{U} / \mathrm{ml}$ to $15.3 \pm 4.4 \mu \mathrm{U} / \mathrm{ml} ; P$ $=0.05)$ (Fig. 4). Coronary blood flow did not change (115 \pm 9

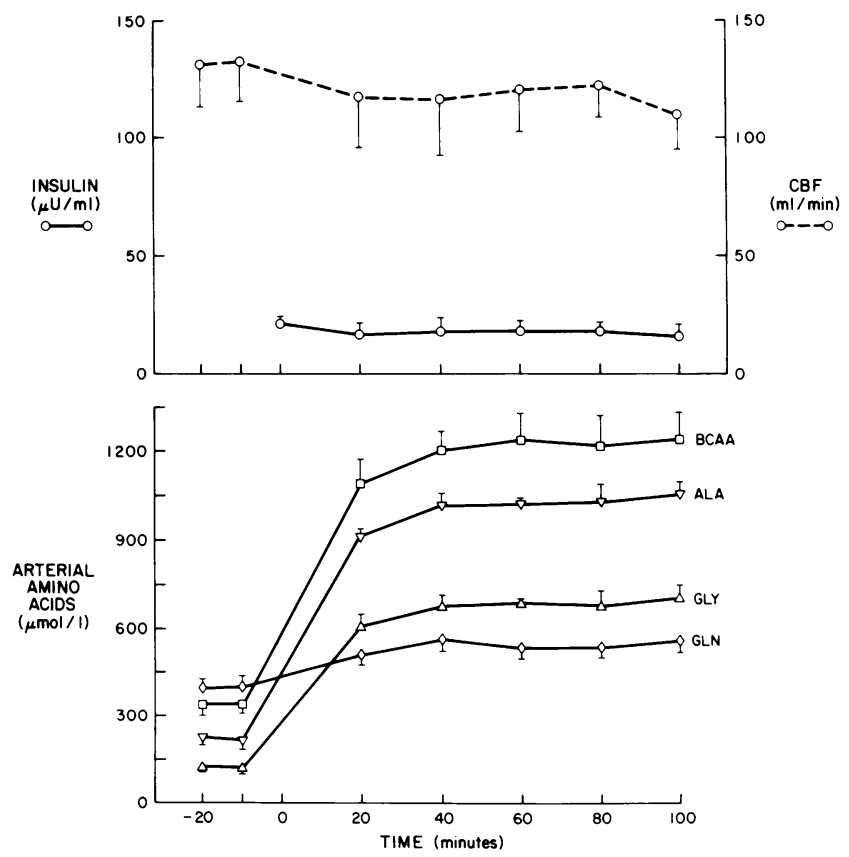

Figure 4. Arterial concentrations of insulin and amino acids and coronary blood flow (CBF) during acute suppression of insulin with somatostatin during amino acid infusion in normal, conscious group III dogs $(n=6)$. Abbreviations are as designated in Fig. 1. 
vs. $103 \pm 10 \mathrm{ml} / \mathrm{min})$. Arterial glucose remained within $10 \%$ of basal levels $(97 \pm 5-88 \pm 2 \mathrm{mg} / \mathrm{dl})$ and myocardial glucose uptake during the steady state infusion (last $40 \mathrm{~min}$ ) was unchanged $(5.6 \pm 0.6 \mu \mathrm{mol} / \mathrm{min})$. In this group, arterial BCAA increased fourfold, alanine and glycine increased fivefold, while glutamine increased to a lesser degree (Table I, Fig. 4). Despite the decline in plasma insulin, there was marked stimulation in myocardial uptake of all BCAA (Table II). Alanine uptake was again observed, but the change from basal was not statistically significant. Myocardial glutamine release did not change.

In Fig. 5, the mean stimulated values of arterial concentration and of net myocardial amino acid balance in the three groups of dogs are summarized. The parallel of arterial concentration and myocardial uptake of the respective amino acid is apparent for each of the BCAA. A similar but less impressive pattern is noted for alanine. This pattern of augmented myocardial uptake with increasing arterial concentrations was not found for any of the other amino acids analyzed. In contrast, augmented insulin concentrations did not result in parallel increases in BCAA uptake. In group III, where insulin levels during the amino acid infusion were the lowest of all groups, arterial concentrations and myocardial uptake of the BCAA were the highest.

Arterial concentrations of each of the BCAA and of total
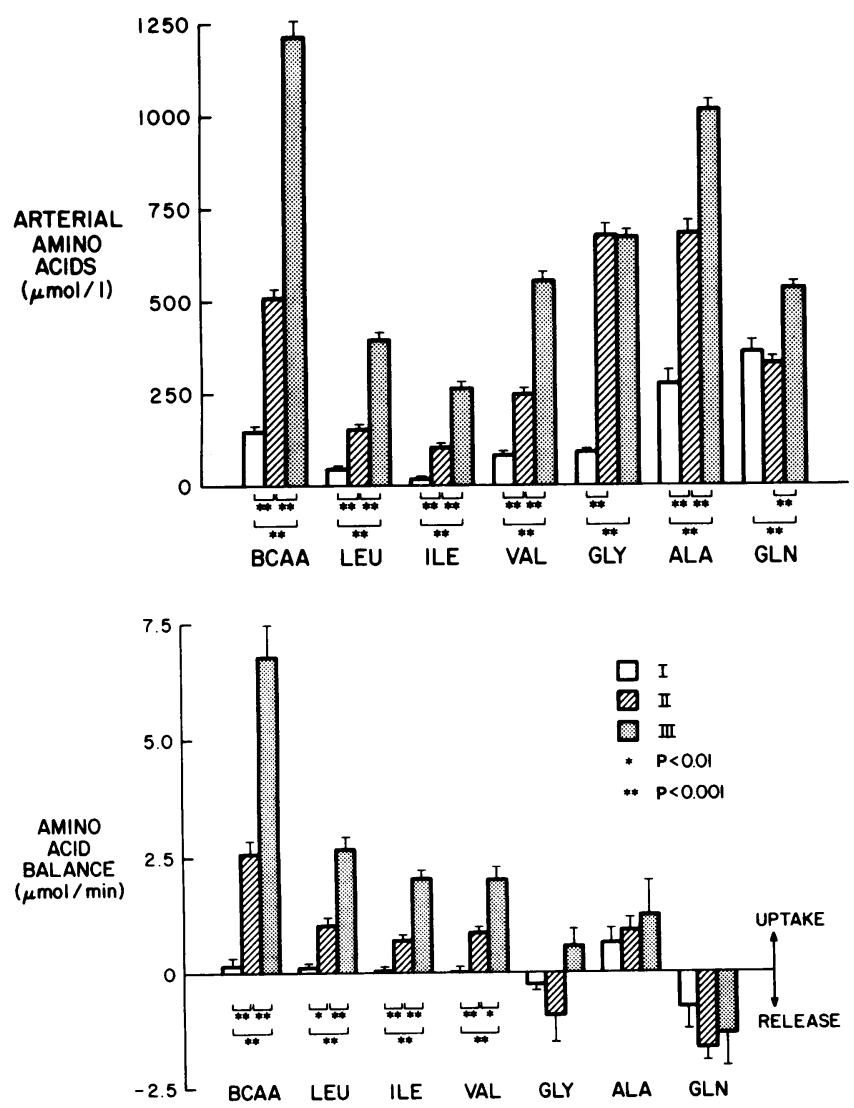

Figure 5. Summary of arterial concentrations (top) and myocardial amino acid balance (bottom) for the amino acids that were found to exhibit a net myocardial flux. Each bar represents the meaned stimulated value of duplicate samples at 60,80 , and $100 \mathrm{~min}$. Group I is represented by the open bars, group II by the cross-hatched bars, and group III by the stippled bars. Abbreviations are as designated in Fig. 1.
BCAA are plotted with their respective myocardial uptakes in Fig. 6. Each point in the figures represents the mean uptake during the last $40 \mathrm{~min}$ (steady state) of an infusion protocol.
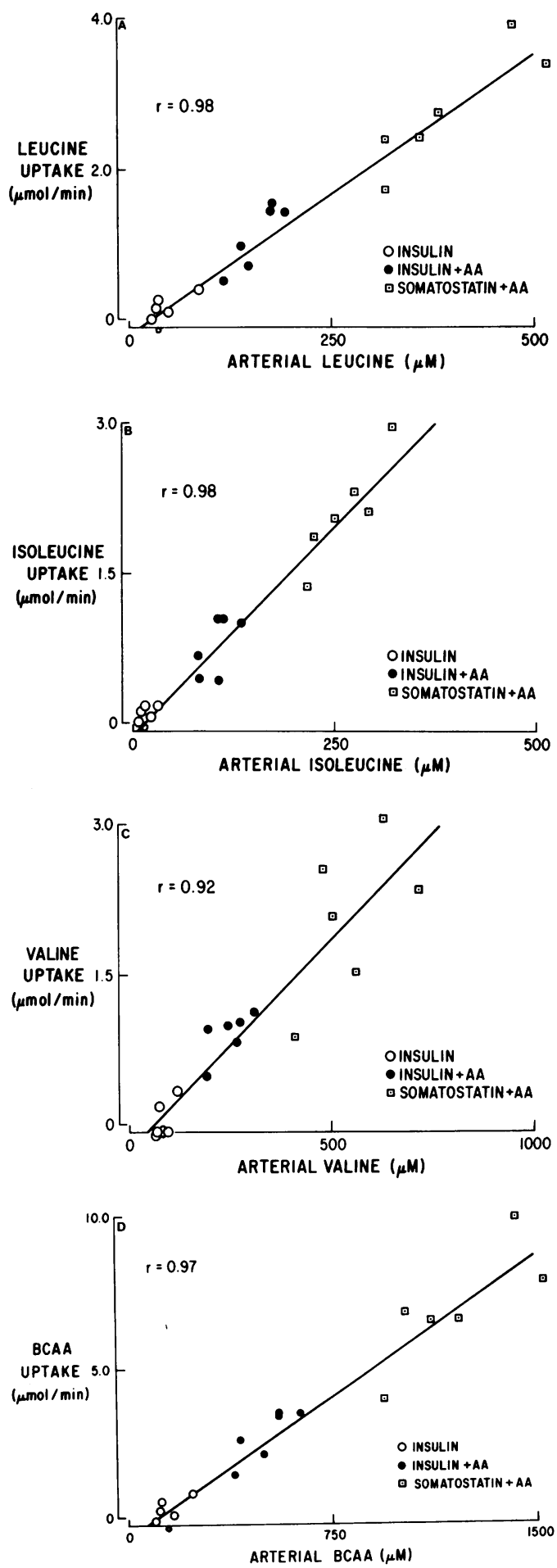

Figure 6. Arterial concentration vs. myocardial uptake of the individual BCAA are illustrated for leucine $(A)$, isoleucine $(B)$, valine $(C)$, and total BCAA $(D)$. All points represent the meaned values during the respective infusion protocol at $60-100 \mathrm{~min}$. The open circles correspond to group I dogs, the closed circles correspond to group II dogs, and the dotted squares represent group III dogs. Correlation coefficients are represented by the $r$ values. 
A correlation coefficient of 0.97 was found for the relationship between arterial concentrations and myocardial uptake of the total BCAA. Highly significant $(P<0.001)$ linear correlation coefficients were found for each of the BCAA: $r=0.98$ for leucine, 0.98 for isoleucine, and 0.92 for valine. This strong linear relationship was found for each of the BCAA even if the hypoinsulinemic dogs (group III) were excluded from analysis. Thus, for the 12 dogs in groups I and II, correlation coefficients were found of 0.96 for total branched chain amino acids, 0.95 for leucine, 0.91 for isoleucine, and 0.95 for valine $(P<0.001$ for each). In contrast, no significant relationship was found in the steady state between arterial concentration and myocardial uptake of alanine $(r=0.32, P>0.10)$.

\section{Discussion}

The current study indicates that $(a)$ in the postabsorptive state, glutamine is the major amino acid involved in net nitrogen transfer out of myocardial tissue; $(b)$ to a lesser extent, alanine in the basal state serves a similar function; $(c)$ in the postabsorptive state and during infusion of amino acids, the BCAA are the principal amino acids taken up by heart muscle, and their circulating concentration largely determines the rate of myocardial uptake; and $(d)$ insulin at physiologic concentrations stimulates heart alanine uptake.

During the basal period, the pattern of myocardial amino acid release is similar to that seen for human forearm muscle (26), where alanine and glutamine function as the principal substrates involved in the transfer of nitrogen from peripheral muscle to splanchnic tissues. As in skeletal muscle, the dominance of myocardial alanine and glutamine release relative to their abundance in muscle protein argues for their de novo synthesis (3). Pyruvate derived from glucose is the likely source of alanine, whereas a number of substrates contributing carbon to the tricarboxylic acid cycle can serve as precursors for alpha-ketoglutarate and subsequently glutamate and glutamine. Nitrogen uptake from ammonia and retention in glutamine by glutamine synthetase in perfused rabbit septum has been recently demonstrated with the use of labeled ammonia $\left({ }^{13} \mathrm{NH}_{3}\right)$ and positron emission tomographic techniques (27), and may be an important source of nitrogen for glutamine release that was observed in the current study.

Our finding of significant net basal myocardial flux of the BCAA, glycine, alanine, and glutamine in the conscious, postabsorptive dog is at variance with results of studies of myocardial balance in man $(9-11)$. However, as previously noted, results of these human studies have not provided consistent descriptions of myocardial amino acid balance. The experimental basis for differing results obtained in these human studies is not obvious. Altered amino acid metabolism secondary to large or small vessel ischemic disease may provide a partial explanation. In this regard, studies of isolated rabbit papillary muscle have documented alanine production and glutamate depletion during periods of anaerobic metabolism $(28,29)$.

The release of glutamine and uptake of BCAA that we observed during the basal postabsorptive period are also findings not consistently observed in human studies. This difference may reflect inter-species variation. However, with regard to glutamine, the use in the current study of a recently validated method for glutamine analysis (30) may contribute to the difference of results. It is worth noting that although the chromatographic procedure used in their study limited the resolution of glutamine, Brodan et al. (11) reported that a combination of glutamine and asparagine was released by the ischemic human heart. These same authors observed uptake of leucine and isoleucine by human myocardium during pacing stress.

A striking finding of the current study was the dependence of myocardial BCAA uptake upon the arterial concentration of these substrates and the seeming lack of effect of insulin on myocardial BCAA balance. Upon further examination of the data in Fig. 6, it is apparent that while myocardial uptake of each of the BCAA increases linearly with increasing amino acid concentration, the slope of the relationship is greater for either isoleucine or leucine than for valine $(P<0.001$ for each comparison). Interestingly, in the isolated perfused heart, raising the concentration of all amino acids in the perfusate by fivefold led to a 3-5-fold increase in the rate of uptake of leucine and isoleucine but not of other amino acids $(3,4)$. This augmentation of uptake of leucine and isoleucine occurred in the absence of insulin in the heart perfusate.

The observation that net myocardial BCAA uptake occurs both in the basal state when plasma insulin is low, and was greatest in the group III dogs with suppressed insulin levels, suggests that hyperinsulinemia is not required to stimulate heart uptake of BCAA. We cannot, on the basis of these studies, exclude the possibility that there is a permissive effect of a critical, low level of insulin in facilitating myocardial BCAA uptake. However, within the physiologic concentrations we tested, insulin did not affect myocardial uptake of the BCAA. It is of interest that in group III, dogs arterial amino acid levels were higher than in group II (Fig. 5). Since both groups of dogs received identical amino acid infusions, it is likely that hyperinsulinemia in group II dogs stimulated disposal of infused amino acids. This effect of insulin was particularly apparent for the BCAA, and is consistent with the known action of insulin in promoting BCAA uptake by skeletal muscle. Thus, in response to the physiologic stimulus of insulin in vivo, it is likely that skeletal muscle takes up BCAA more readily than does myocardium. However, we emphasize that these results do not necessarily indicate that the stimulatory effects of insulin on protein synthesis are less prominent in heart than in skeletal muscle.

The striking linear correlation of myocardial uptake of each of the BCAA with their plasma level suggests that factors that decrease the arterial BCAA concentrations (e.g., insulin administration, or glucose ingestion) may actually decrease heart BCAA uptake. The trend towards decline in BCAA uptake in group I dogs supports this suggestion. On the other hand, a rise in plasma BCAA, as occurs after a protein meal, would be expected to increase heart BCAA uptake. In this regard, the BCAA levels seen in group II dogs are typical of those seen in post-cibal man $(31,32)$.

In the current study, the cellular fate of extracted BCAA within myocardial tissue was not directly examined. Four general pathways of further metabolism might be considered: (a) accumulation of the free amino acids within the cytosol; (b) incorporation into heart protein; (c) transamination of the BCAA to their keto-acid analogues which are then released into the circulation; and $(d)$ oxidation of the $\mathrm{BCAA}$ to $\mathrm{CO}_{2}$ and water. Precise definition of the relative contribution of each of these pathways to myocardial BCAA metabolism would require a combination of radiotracer and balance mea- 
surements that go well beyond those undertaken in the current study. However, based upon our amino acid balance measurements and data available from studies in the perfused heart, we consider that pathway $c$ and/or $d$ are the most likely to play a major quantitative role. This consideration is grounded upon the following observations. In our group III animals, uptake of each of the BCAA averaged between 2.0 and 2.7 $\mu \mathrm{mol} / \mathrm{min}$ over the last $40 \mathrm{~min}$ of the study. The dog hearts in our study averaged $150 \mathrm{~g}$ wet weight and would be expected to have a cell water volume of $\sim 84 \mathrm{ml} \mathrm{(33)}$. Simple accumulation of the BCAA within cell water would result in a rise in cell concentration to $1.1 \mathrm{mmol}$ for each, even if only the amount taken up over the last $40 \mathrm{~min}$ is considered. This is 2-4 times higher than the simultaneously measured arterial concentration of each of the BCAA (Table I). Morgan et al. (4) have previously shown that in the rat heart in vivo, the cytosolic concentration of the BCAA is slightly less than the serum concentration, and when rat heart is perfused with an amino acid mixture containing BCAA at a concentration fivefold greater than in vivo, the cytosolic BCAA concentration remains at $\sim 20-25 \%$ of the perfusate. Furthermore, studies of the sarcolemma amino acid transporting systems of heart muscle indicate that the BCAA, unlike some neutral amino acids, enter the myocyte principally via the L-System $(34,35)$. Unlike System A and ASC, System L shows no sodium dependence and does not transport amino acids against a concentration gradient. Therefore, we regard it as unlikely that simple cytosolic accumulation of BCAA contributes more than a very modest amount to total myocardial BCAA disposal.

Some of the BCAA removed by the heart are almost certainly being incorporated into protein. It is noteworthy that in the isolated perfused heart, leucine added to the perfusate is particularly effective in stimulating protein synthesis (36). However, since we observed no net uptake of the essential amino acids phenylalanine or methionine or of other amino acids that might have been incorporated into nascent heart protein along with the BCAA (Table II), we consider it unlikely that a major fraction of the extracted BCAA were incorporated into protein. Rigorous quantification of the contribution of protein synthesis to BCAA disposal would require measurements of the rates of protein synthesis using isotope incorporation methods similar to those used extensively in the isolated perfused heart (3).

There is little information in the literature which would allow us to discern whether direct release of the keto acid analogues of the BCAA contributes significantly to the disposal of extracted BCAA. It is known that in postabsorptive man, skeletal muscle releases the keto-acid counterparts of leucine and valine (alpha-ketoisocaproate and alpha-ketoisovalerate, respectively). However, when amino acids are infused in man and skeletal muscle BCAA uptake is stimulated there is no significant change in muscle release of BCAA keto-acid derivatives (26). Resolution of the contribution of ketoacids to the disposal of BCAA by the heart must await quantitation of their balance in vivo.

The final possible fate of the extracted BCAA would be oxidation. Myocardium can use amino acids as an oxidative fuel (3), and the BCAA appear particularly suitable substrates for heart energy metabolism (37). It is of interest that both production of keto-acid derivatives and BCAA oxidation, which appear to be the two most likely fates of infused BCAA, involve loss of the alpha-amino nitrogen. In skeletal muscle, alanine, glutamine, and ammonia are thought to be important mediators in the transport of amino groups from muscle to splanchnic tissues $(7,38)$. In the current study there was no increase in release of glutamine, alanine, or other amino acids above basal even when BCAA uptake was maximal (group III). Indeed, in each of the three experimental groups, alanine was removed by the heart during the infusion period. Whether myocardial ammoniagenesis increases during amino acid infusion is unknown, and it will require arterial-coronary sinus measurements of ammonia to resolve this question.

The significant increase in myocardial alanine uptake seen in groups I and II could be explained by the effect of insulin to increase pyruvate dehydrogenase activity. An effect of insulin on myocardial pyruvate dehydrogenase might occur directly on enzyme activity or indirectly from decreased availability of fatty acids for tricarboxylic acid cycle oxidation. We have previously observed an increase in heart lactate uptake during physiologic hyperinsulinemia (13), which may occur by similar mechanisms.

The findings of the current study provide physiologic data which have implications concerning the use of radionuclide imaging to assess myocardial metabolic events. The advent of positron and single photon computed tomography and the production of radiolabeled carbon-11, fluorine-18, and N-13 labeled branched chain amino acid metabolites may permit direct assessment of regional myocardial metabolic function $(20,39,40)$. Awareness of the effects of a varying metabolic milieu upon myocardial utilization of these substrates is critical for evaluating measurements of altered metabolism under pathologic conditions.

\section{Acknowledgments}

We wish to thank Frank Pelletier and Lee Surkin for technical assistance and Dr. R. Hendler for performing the insulin immunoassay.

Dr. Schwartz was recipient of U. S. Public Health Service (USPHS) National Research Service Award HL-06744-01 (1983-1984), and currently is recipient of American Heart Association (Connecticut Affiliate, Fairfield Chapter) Grant-in-Aid 11-213-845. Dr. Barrett is recipient of USPHS SERCA-Diabetes Mellitus/Cardiovascular grant AM00888. This work was supported in part through AHA grant 831264.

\section{References}

1. Krebs, H. A. 1972. Some aspects of the regulation of fuel supply in omnivorous animals. Adv. Enzyme Regul. 10:397-420.

2. Bing, R. J. 1965. Cardiac metabolism. Physiol. Rev. 45:171202.

3. Morgan, H. E., D. E. Rannels, and E. E. McKee. 1979. Protein metabolism of the heart. In Handbook of Physiology. The Cardiovascular System. I. American Physiological Society, Bethesda. 845-871.

4. Morgan, H. E., D. C. Earl, A. Broadus, E. B. Wolpert, K. E. Giger, and L. S. Jefferson. 1971. Regulation of protein synthesis in heart muscle. I. Effect of amino acid levels on protein synthesis. $J$. Biol. Chem. 246:2152-2162.

5. Morgan, H. E., E. S. Jefferson, E. B. Wolpert, and D. E. Rannels. 1971. Regulation of protein synthesis in heart muscle. II. Effect of amino acid levels and insulin on ribosomal aggregation. J. Biol. Chem. 246:2163-2170.

6. Chua, B. H., D. L. Siehl, and H. E. Morgan. 1980. A role for leucine in regulation of protein turnover in working rat hearts. Am. J. Physiol. 239:E510-E514.

7. Goldberg, A. L., and T. W. Chang. 1978. Regulation and 
significance of amino acid metabolism in skeletal muscle. Fed. Proc. 37:2301-2307.

8. Pain, V. M., and P. J. Garlick. 1974. Effect of streptozotocin diabetes and insulin treatment on the rate of protein synthesis in tissues of the rat in vivo. J. Biol. Chem. 249:4510-4514.

9. Carlsten, A., B. Hallgren, R. Jagenburg, A. Svanborg, and L. Werko. 1961. Myocardial metabolism of glucose, lactic acid, amino acids and fatty acids in healthy individuals at rest and at different work loads. Scand. J. Clin. Invest. 13:418-428.

10. Mudge, G., R. Mills, H. Taegtmeyer, R. Gorlin, and M. Lesch. 1976. Alterations of myocardial amino acid metabolism in chronic ischemic heart disease. J. Clin. Invest. 58:1185-1192.

11. Brodan, V., J. Fabian, M. Andel, and J. Pechar. 1978. Myocardial amino acid metabolism in patients with chronic ischemic heart disease. Basic Res. Cardiol. 73:160-170.

12. DeFronzo, R., J. Tobin, and R. Andres. 1979. The glucose clamp technique. Am. J. Physiol. 237:E214-E223.

13. Barrett, E. J., R. G. Schwartz, C. K. Francis, and B. L. Zaret. 1984. Regulation by insulin of myocardial glucose and fatty acid metabolism in the conscious dog. J. Clin. Invest. 74:1073-1079.

14. Lazar, H. L., G. D. Buckberg, A. J. Manganaro, H. Becker, and J. V. Maloney, Jr. 1980. Reversal of ischemic damage with amino acid substrate enhancement during reperfusion. Surgery. 88:702-709.

15. Rau, E. E., K. I. Shine, A. Gervais, A. M. Douglas, and E. C. Amos III. 1979. Enhanced mechanical recovery of anoxic and ischemic myocardium by amino acid perfusion. Am. J. Physiol. 236(6):H873H879.

16. Pisarenko, O. I., E. S. Solomatina, I. M. Studneva, V. E Ivanov, V. I. Kapelko, and V. N. Smirnov. 1983. Effect of glutamic and aspartic acids on adenine nucleotides, nitrogenous compounds and contractile function during underperfusion of isolated rat heart. $J$. Mol. Cell. Cardiol. 15:53-60.

17. Bittl, J. A., and K. I. Shine. 1983. Protection of ischemic rabbit myocardium by glutamic acid. Am. J. Physiol. 245:H406-H412.

18. Shine, K., J. Krivokapich, A. Douglas, and J. Barrio. 1983. Biochemical basis for improved mechanical recovery of mammalian myocardium with glutamate. Circulation. 68:III-68. (Abstr.)

19. Uretzky, G., I. Vinograd, H. Freund, E. Yaroslavsky, Y. J. Applebaum, and J. B. Borman. 1984. The protective effect of branched chain amino acids in myocardial ischemia. J. Am. Coll. Cardiol. 3: 608. (Abstr.)

20. Barrio, J. R., F. J. Baumgartner, E. Henze, M. S. Stauber, J. E. Egbert, N. S. MacDonald, H. R. Schelbert, M. E. Phelps, and F. T. Liu. 1983. Synthesis and myocardial kinetics of $\mathrm{N}-13$ and $\mathrm{C}-11$ labeled branched chain L-amino acids. J. Nucl. Med. 24:937-944.

21. Gelbard, A. S., L. P. Clarke, J. M. McDonald, W. G. Monahan, R. S. Tilbury, T. Y. T. Kuo, and J. S. Laughlin. 1975. Enzymatic synthesis and organ distribution studies with ${ }^{13} \mathrm{~N}$-labeled L-glutamine and L-glutamic acid. Radiology. 116:127-132.

22. Bergmeyer, H. U., and E. Bernt. 1974. Determination with glucose oxidase and peroxidase. In Methods of Enzymatic Analysis, Vol. 3. H. Bergmeyer, editor. 1205-1212.

23. Ganz, W., K. Tamura, H. Marcus, R. Donoso, S. Yoshido, and H. Swan. 1971. Measurement of coronary sinus blood flow by continuous thermodilution in man. Circulation. 44:181-195.
24. Pepine, C., J. Mehta, W. Webster, and W. Nichols. 1978. In vivo validation of a thermodilution method to determine regional left ventricular blood flow in patients with coronary disease. Circulation. 58:795-802.

25. Snedecor, G. W., and W. G. Cochran. 1980. Statistical Methods. Iowa State University Press, Ames, IA. 255-269.

26. Abumrad, N. N., D. Rabin, K. L. Wise, and W. W. Lacy. 1982. The disposal of an intravenously administered amino acid load across the human forearm. Metab. Clin. Exp. 31:463-470.

27. Krivokapich, J., J. B. Barrio, M. E. Phelps, C. R. Watanabe, R. E. Keen, H. C. Padgett, A. Douglas, and K. I. Shine. 1984. Kinetic characterization of ${ }^{13} \mathrm{NH}_{3}$ and $\left[{ }^{13} \mathrm{~N}\right]$ glutamine metabolism in rabbit heart. Am. J. Physiol. 246:H267-H273.

28. Taegtmeyer, H., A. G. Ferguson, and M. Lesch. 1977. Protein degradation and amino acid metabolism in autolyzing rabbit myocardium. Exp. Mol. Pathol. 26:52-62.

29. Taegtmeyer, H., M. B. Peterson, V. R. Ragavan, A. G. Ferguson, and M. Lesch. 1977. De novo alanine synthesis in isolated oxygen deprived rabbit myocardium. J. Biol. Chem. 252:5010-5018.

30. Jacob, R., and E. J. Barrett. 1982. Chromatographic analysis of glutamine in plasma. J. Chromatogr. 229:188-192.

31. Wahren, J., P. Felig, and L. Hagerfeldt. 1976. Effect of protein ingestion on splanchnic and leg metabolism in normal man and in patients with diabetes mellitus. J. Clin. Invest. 57:987-999.

32. Cahill, G. F., Jr. 1976. Protein and amino acid metabolism in man. Circ. Res. 38(Suppl. I):109-114.

33. Scharff, R., and I. G. Wool. 1965. Accumulation of amino acids in muscle of perfused rat heart: effect of insulin. Biochem. J. 97: 257-271.

34. Gazzola, G. C., R. Franchi, V. Saibene, P. Ronchi, and G. G. Guidotti. 1972. Regulation of amino acid transport in chick embryo heart cells. I. Adaptive system of mediation of neutral amino acids. Biochim. Biophys. Acta. 266:407-421.

35. Guidotti, G. G., A. F. Borghetti, and G. C. Gazzola. 1978. Regulation of amino acid transport in animal cells. Biochim. Biophys. Acta. 515:329-366.

36. Ichihara, K., J. R. Neely, D. L. Siehl, and H. E. Morgan. 1980. Utilization of leucine by the working rat heart. Am. J. Physiol. 239: E430-E436.

37. Buse, M. G., J. F. Biggers, K. H. Friderici, and J. F. Buse. 1972. Oxidation of branched chain amino acids by isolated hearts and diaphragms of the rat. The effect of fatty acids, glucose, and pyruvate respiration. J. Biol. Chem. 247:8085-8096.

38. Lowenstein, J. M., and M. N. Goodman. 1978. The purine nucleotide cycle in skeletal muscle. Fed. Proc. 37:2308-2312.

39. Berman, S. R., R. A. Lerch, K. A. A. Fox, P. A. Ludbrook, M. J. Welch, M. M. Ter-Pogossian, and B. E. Sobel. 1982. Temporal dependence of beneficial effects of coronary thrombolysis characterized by positron tomography. Am. J. Med. 73:573-581.

40. Marshall, R. C., J. H. Tillisch, M. E. Phelps, S. C. Huang, R. Carlson, E. Henze, and H. R. Schelbert. 1983. Identification and differentiation of resting myocardial ischemia and infarction in man with positron computed tomography ${ }^{18} \mathrm{~F}$-labelled fluorodeoxyglucose and N-13 ammonia. Circulation. 67:766-778. 